\title{
A comparison of the transillumination- assisted technique versus midline approach technique in novices: a prospective randomized controlled trial about the Bonfils intubation fiberscope
}

\author{
Jian Wang ${ }^{\dagger}$, Lan Yuan ${ }^{\dagger}$, Guoqiang Fu, Wei Tang, Guijie Yu, Feng Guo and Jiangang Song*
}

\begin{abstract}
Background: The present study aimed to compare the safety and efficacy for novices to conduct intubation with the Bonfils intubation fiberscope (BIF) using the transillumination-assisted or midline approach technique in patients with normal airways.

Methods: In this prospective randomized control study, 10 trainees were assigned to the transillumination-assisted technique group (T group) or the midline approach technique group (R group). Each trainee was required to conduct intubation in 50 patients. The primary outcome was intubation time. The secondary outcomes were success rate (\%), number of attempts, and complications.

Results: Among the cases of successful intubation, the intubation time was not significantly different between the two groups $(P>0.05)$. The overall success rate of intubation was not significantly different between the two groups $(P>0.05)$. The intubation success rates at the first, second, and third attempts as well as the average intubation times were similar between the two groups $(P>0.05)$, but in patients receiving successful intubation at the second attempt, the intubation time was longer in the T group $(P=0.0006)$. The incidences of dry throat, sore throat, and hoarseness were higher in the T group (all $P<0.05$ ).
\end{abstract}

Conclusions: For patients with a normal airway, the transillumination-assisted technique was unlikely to increase the success rate of intubation with the BIF compared with the midline approach technique, but led to more complications.

Trial registration: ChiCTR-INR-16009967, retrospectively registered on November 22, 2016

Keywords: Bonfils intubation fiberscope, Tracheal intubation, Transillumination-assisted technique

\section{Background}

Establishing a successful endotracheal intubation is important for pulmonary ventilation and gas exchange during anesthesia and critical care. Failed or difficult endotracheal intubation is an important cause of morbidity and mortality during anesthesia [1]. A variety of methods and tools (such as direct laryngoscope, video laryngoscopes, and fiber optic bronchoscope (FOB))

\footnotetext{
* Correspondence: songjg1993@126.com

${ }^{\dagger}$ Equal contributors

Department of Anesthesiology, Shanghai Shuguang Hospital, Shanghai

University of Traditional Chinese Medicine, Shanghai 201203, China
}

have been used for endotracheal intubation, but no single device or method can be applied to all airway scenarios. Therefore, anesthesiologists have to master a variety of airway devices.

Among these devices, the Bonfils intubation fiberscope (BIF) is widely used. The BIF is a rigid, long, slender device with a curved tip. A tracheal tube is loaded onto the shaft of the BIF, inserted into the mouth, and advanced to the glottis aperture. After identifying the vocal cords, the tracheal tube is placed into the trachea $[2,3]$. The BIF can be used for the endotracheal intubation normal airways, but is especially useful for difficult tracheal 
intubations for patients with limited cervical mobility or limited mouth opening $[4,5]$. The most important disadvantage of the BIF is the long learning curve $[2,6]$.

Two methods are commonly used for targeting the glottis using BIF: the midline approach technique and the transillumination-assisted technique. The midline approach technique is one of the most common techniques for BIF; the operators directly observe through the eyepieces to look for the glottis under direct vision [6], intra-oral mist, secretions, or contamination impair the vision during intubation and are the main reasons for BIF intubation failure. Recent studies showed that the application of the transilluminationassisted technique can improve the success rate of intubation [7]. In the transillumination-assisted technique, an external light is used to locate the glottis through the cricothyroid membrane [8]. Since the transillumination-assisted technique does not entirely rely on the direct observation of the glottis through the eyepieces, it is less likely to be affected by blurred vision.

Nevertheless, which technique is easier for trainees remain to be shown. Therefore, we hypothesized that the transillumination-assisted technique have a higher success rate for intubations performed by trainees. Therefore, this study compared the transillumination-assisted and midline approach techniques in terms of intubation time, success rate, and intubation-related complications, hence providing clinical data to further optimize the learning and training process of BIF-guided tracheal intubation.

\section{Methods}

\section{Study design}

This prospective randomized controlled trial was conducted at the Anesthesiology Department of Shuguang Hospital Affiliated to Shanghai University of Traditional Chinese Medicine, the study was approved by the ethics committee of the hospital. The trial is registered with Clinicaltrials.gov (ChiCTR-INR-16009967), available online: http://www.chictr.org.cn/showproj.aspx?proj=15963.

Between January 2013 and June 2014, 500 patients undergoing tracheal intubation were recruited. Tracheal intubation operations were conducted by 10 students (trainees) from the Anesthesiology Department of Shuguang Hospital Affiliated to Shanghai University of Traditional Chinese Medicine. These trainees had $\leq 2$ years of doctor's qualification, but did not have experience with the BIF. They were assigned to the transilluminationassisted technique group ( $\mathrm{T}$ group) or the midline approach technique group ( $\mathrm{R}$ group) using a simple randomization method based on a random number table.

The trainees were provided standard training by a chief physician who was considered as the observer and was skilled in using the Bonfils optical stylet (>100 successful unsupervised intubation operations).
The training included explaining the theory of the BIF technique, watching teaching videos, and guiding the trainees to conduct tracheal intubation using the BIF in manikins (Shanghai Xinman Co., Ltd., Shanghai, China) [9]. After the trainees had successfully conducted tracheal intubation in manikins for three times, they were randomized and performed intubation operations.

\section{Patients}

The inclusion criteria were: 1) patients undergoing elective surgery under general anesthesia; 2) age 18-65 years; 3) ASA grade I or II; 4) Mallampati grade I-II; 5) body mass index (BMI) of $18-25 \mathrm{~kg} / \mathrm{m}^{2}$; and 6) signed the written informed consent. The exclusion criteria were: 1) severe cardiopulmonary system diseases; 2 ) coagulation disorders; 3) missing teeth or removable denture; 4) obesity; or 5) difficult airway.

\section{Blinding}

Since this test could not be blind to observers and operators, only the patients were blinded to the kind of intubation.

\section{Preparation prior to intubation}

The BIF stylet (Karl Storz, Tuttlingen, Germany) was connected to the light source, the objective lens was cleaned with alcohol, and the focal length of the eyepiece was adjusted. The shaft was lubricated with paraffin oil and nested with a suitable type of tracheal tube whose front end was $0.5 \mathrm{~cm}$ beyond the front end of the lens shaft; 7.5- and 7-mm tracheal tubes were used for male and female patients, respectively.

Prior to operation, the patients were fasted for $8 \mathrm{~h}$ and did not receive any medication. In the operation room, the patient was placed in a supine position, and the venous pathway was opened. Blood pressure, heart rate, electrocardiogram, oxygen saturation, and trainof-four stimulation (TOF) were routinely monitored. The bispectral index (BIS) was monitored using an ASPECT 2000 monitor (Aspect A-2000, MA, USA). The patient was given preoxygenation at $8-10 \mathrm{~L} / \mathrm{min}$ for $3 \mathrm{~min}$, followed by anesthetic induction with an intravenous injection of $0.05 \mathrm{mg} / \mathrm{kg}$ of midazolam, 2 $\mu \mathrm{g} / \mathrm{kg}$ of fentanyl, $2 \mathrm{mg} / \mathrm{kg}$ of propofol, and $0.6 \mathrm{mg} / \mathrm{kg}$ of rocuronium, as well as tracheal intubation after the BIS value was $40-60$ and TOF indicated the disappearance of $\mathrm{T} 2$.

\section{Tracheal intubation with transillumination-assisted technique}

The head of the patient was maintained in a neutral position without flexion and extension movements. The operator stood near the left side of the patient's head. 
Then, the operator lifted the jaw of the patient with his left hand and inserted the optical laryngoscope into the mouth along the incisor midline with the right hand. The operator would look for the brightest transilluminated spot to identify the glottis (Fig. 1). The stylet position was identified under the eyepiece: if it was located at the superior glottis or entered the trachea, the tracheal tube was inserted into the trachea along the lens shaft. However, if the glottis or tracheae ring was invisible, the optical stylet position was adjusted again to continuously look for the brightest spot at the neck midline, and the aforementioned procedures were repeated [7] (Fig. 1).

\section{Tracheal intubation via midline approach technique}

The operator directly observed through the eyepieces to adjust the depth and the direction of the lens body, thereby to look for the glottis and insert the tracheal tube into the trachea along the lens body under direct vision. After successful tracheal intubation, the optical stylet was retracted along the physiological curvature of the oropharynx.

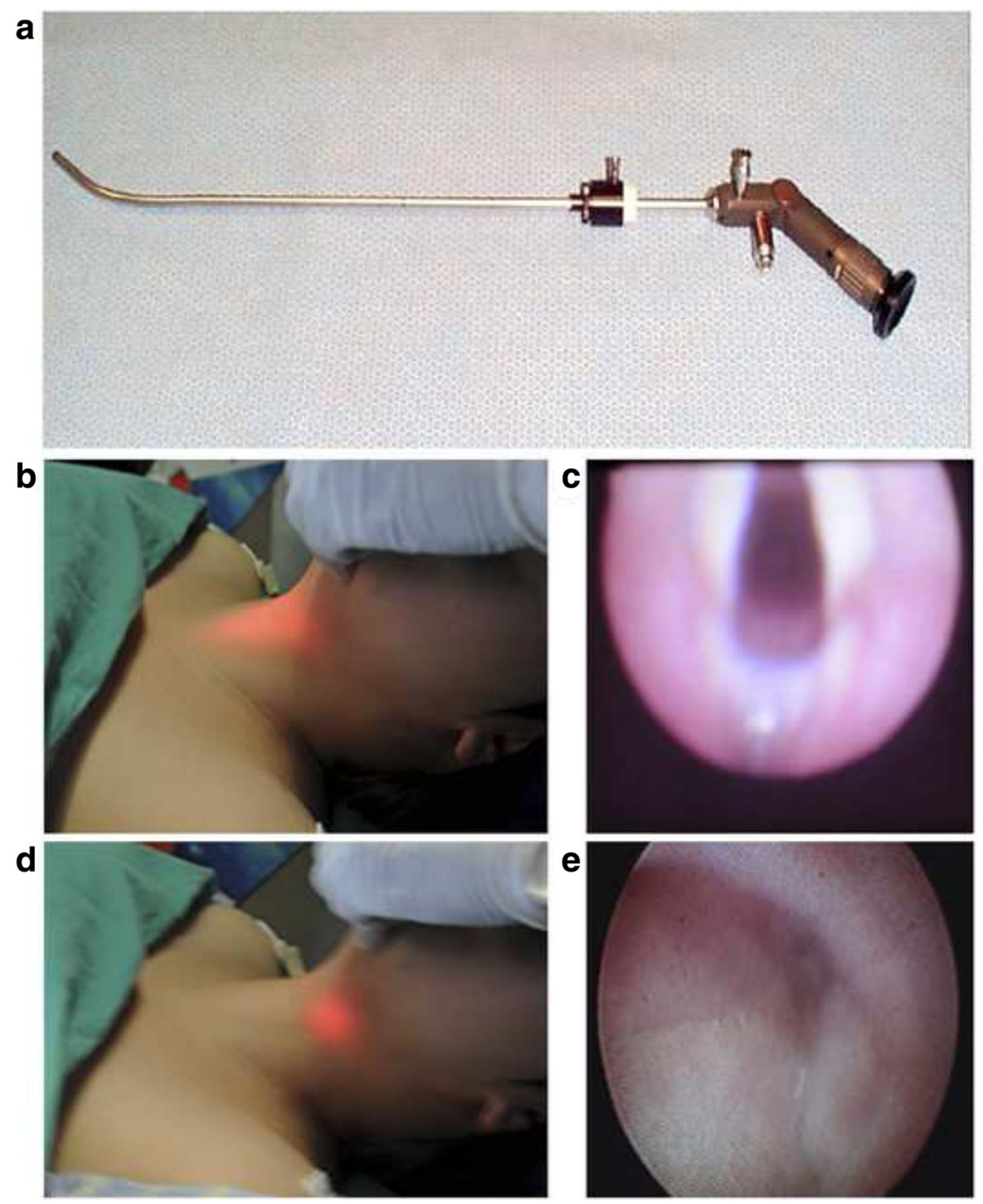

Fig. 1 Transillumination-assisted technique and midline approach technique. Note: In intubation using the transillumination-assisted technique, the Bonfils optical stylet (a) with a preset tracheal tube was slowly inserted into the upper respiratory tract along the midline. If a centered bright spot was visible in the cricothyroid membrane region of the neck (b), it might indicate that the laryngoscope tip was located near the glottis, where the vocal cords, annulus tracheae, and other structures were visible (c). If the laryngoscope tip was deviated to one side, the spot was more focal and darker (d), where sinus piriformis, glossoepiglottic folds, and other structures were visible (e) 


\section{Outcomes}

The primary outcome was the intubation time. The secondary outcomes were success rate, number of attempts, and complications.

All intubation operations were observed by the same observer, who was in charge of recording experimental results, to avoid interobserver bias. This observer also recorded the characteristics of the patients, information on operators, and complications. The data included intubation attempts, success rate of first intubation, overall success rate of three attempts of intubation, intubation time, and intubation-related complications.

The tracheal intubation time was defined as the time elapsed from the insertion of the front end of the laryngoscope into the mouth to the retraction of the laryngoscope after tracheal intubation was completed. The total duration for intubating a patient could not be longer than $3 \mathrm{~min}$, and the maximum number of attempts allowed was three. The cases with three unsuccessful attempts or total intubation time $>3$ min were considered intubation failure. In addition, patients with blood oxygenation falling to $<92 \%$ before intubation could be completed were considered as failed. In all these cases, intubation was completed by the observer.

\section{Statistical analysis}

In a preliminary study, the average intubation time was $30.0 \pm 18.4 \mathrm{~s}$ using BIF with the midline approach technique. An intubation time difference of $50 \%$ was considered to be clinically significant. According to the calculation formula of sample size for two independent samples $N=\left[\left(Z \alpha / 2+Z_{\beta}\right) \times \sigma / \delta\right]^{2} \times 4\left(Q_{1}{ }^{-1}+Q_{2}{ }^{-1}\right)$, where type I error alpha value of 0.05 and power of $90 \%$. Thus a sample size of about 500 patients is needed.

Statistical analyses were performed using the SPSS 13.0 software (SPSS Inc., IL, USA). Categorical data was expressed as number or percentage. Continuous data was expressed as mean \pm standard deviation. The categorical data was analyzed using the chi-square test, and the continuous data was analyzed using the Student $t$ test. The linear regression analysis about the intubation time and patient's treatment order were performed. Two-sided $P$-values $<0.05$ were considered statistically significant.

\section{Results}

\section{Subjects characteristics}

A total of 500 ASA grade I or II patients were recruited (Fig. 2). Ten trainees were selected to conduct tracheal intubation (five trainees/group). The general data of the trainees and patients are listed in Table 1. Additional file 1: Figures S1, S2, and S3 present the learning curves of the two groups.

\section{Primary outcome}

The number of attempts was similar between the two groups $(1.4 \pm 0.7$ vs. $1.3 \pm 0.6, P=0.056)$. In patients with successful intubation at the second attempt, the intubation time was longer in the T group (67.4 \pm 23.7 vs. $52.7 \pm 22.1 \mathrm{~s}, P=0.006)$, without difference for successful intubation at the first or third attempt (46.7 \pm 18.1 vs. $48.6 \pm 19.9 \mathrm{~s}, P=0.348 ; 80.7 \pm 19.8$ vs. $63.8 \pm 25.0 \mathrm{~s}$, $P=0.054)$. Among all patients with successful intubation, the intubation time was similar between the two groups $(52.8 \pm 22.2$ vs. $49.9 \pm 20.7$ s, $P=0.137)$. No intubation was failed because of blood oxygenation $<92 \%$.

\section{Secondary outcomes}

The success rates of the first, second, and third attempts were similar between the two groups $(71.6 \%$ vs. $78.0 \%, P=0.100 ; 57.8 \%$ vs. $65.5 \%, P=0.301 ; 58.1 \%$ vs. $57.9 \%, P=0.991)$. In addition, the overall intubation success rate was not significantly different between the two groups (94.8\% vs. $96.8 \%, P=0.265)$.

Among patients receiving intubation with the transillumination-assisted technique, intubation was successful at the first, second, and third attempts in $71.6 \%$ (179/250), 16.0\% (40/250), and 7.2\% (18/250) of patients, respectively. Intubation was unsuccessful after three attempts in $5.2 \%(13 / 250)$ of patients, mainly because the trainees failed to find the bright spot. Among patients receiving intubation with the midline approach technique, intubation was successful at the first, second, and third attempts in $78.0 \%(195 / 250), 14.4 \%(36 / 242)$, and $4.4 \%(11 /$ 242) of patients, respectively. Intubation was unsuccessful after three attempts in $3.2 \%(8 / 250)$ of patients, mainly because the trainees could not clearly observe the glottis.

Compared to the midline approach technique, the frequencies of dry throat (52.2\% vs. $40.4 \%, P=0.020)$, sore throat $(26.8 \%$ vs. $16.8 \%, P=0.009)$ and hoarseness $(14 \%$ vs. $8 \%, P=0.045)$ were higher in the transilluminationassisted technique (Table 2).

\section{Discussion}

This study compared the intubation time, success rate and complications of the BIF conducted by novices using the midline approach technique or the transilluminationassisted technique. It was found that the intubation time and intubation success rate were similar between the two techniques. The intubation time for patients receiving successful intubation at the second attempt was longer with the transillumination-assisted technique than with the midline approach technique. The complication frequency of the transillumination-assisted technique was significantly higher compared to the midline approach technique.

In this study, the overall success rate of intubation with the BIF was $94.8-96.8 \%$. Byhahn et al. reported a success rate of $81.6 \%$ in simulating patients with a 


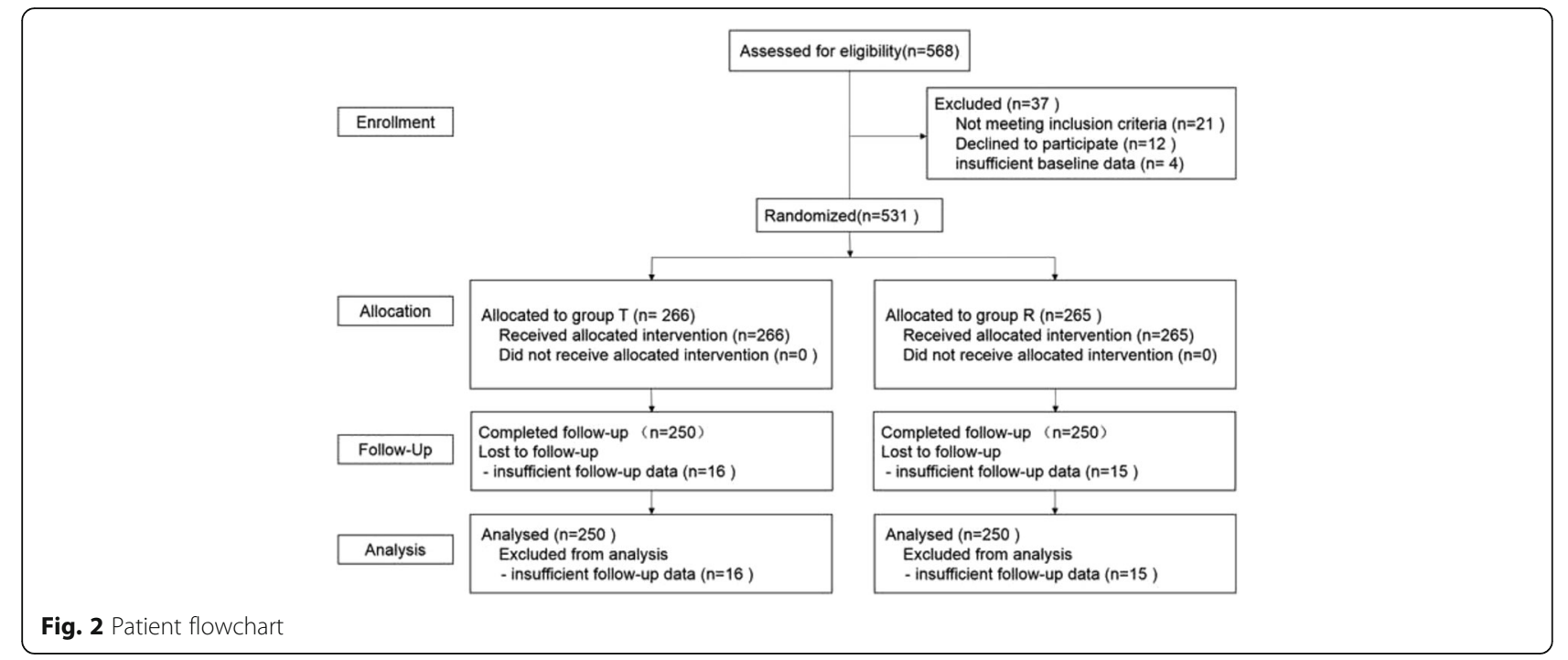

difficult airway who received tracheal intubation with the BIF [10]. Piepho et al. conducted a study of novices using the BIF in a simulated difficult airway and showed that the success rate of the BIF intubation was $82 \%$, while the success rate of BIF intubation was higher than that of Macintosh laryngoscopy for patients with tongue edema ( $84 \%$ vs. $76 \%$ ) [3]. A number of studies reported a success rate of BIF intubation to be $82-100 \%[6,11,12]$. In this study, the success rate of intubation with the transillumination-assisted technique was $97.3 \%$, similar to that reported by Sui [13].

In this study, the average intubation time with the BIF was 50.0-52.8 s, which was close to the upper limit of the time range of $23-52$ s reported previously [6, 12 , 14]. Differences in approach such as left molar approach, para-tongue approach, or modified laryngoscopic structure [15] are possible factors affecting intubation time. Different methods can be used to judge successful intubation, such as the carbon dioxide test or lung auscultation, which may have different durations and lead to different time points, thereby exerting different effects on the results. Some scholars believe that compared to operators who are experienced in conventional laryngoscopy, novices would take shorter time to conduct BIF intubation [2]. When the operators are familiar with the new intubation tools after training, the trainees and

Table 1 Characteristics of trainees and airway data of patients undergoing tracheal intubation using the Bonfils optical stylet

\begin{tabular}{|c|c|c|c|}
\hline & T group $(n=250)$ & R group $(n=250)$ & $P$ value \\
\hline \multicolumn{4}{|l|}{ Characteristics of patients } \\
\hline Gender (male/female) & $137 / 113$ & $133 / 117$ & 0.720 \\
\hline Age (years) & $48.0 \pm 12.6$ & $47.1 \pm 12.6$ & 0.436 \\
\hline Height (m) & $1.68 \pm 0.08$ & $1.67 \pm 0.08$ & 0.207 \\
\hline Weight (kg) & $62.9 \pm 9.0$ & $63.1 \pm 9.0$ & 0.777 \\
\hline $\mathrm{BMI}\left(\mathrm{kg} / \mathrm{m}^{2}\right)$ & $21.8 \pm 2.6$ & $22.2 \pm 2.6$ & 0.123 \\
\hline ASA $1 / 2 / 3$ & $105 / 145 / 0$ & $111 / 139 / 0$ & 0.588 \\
\hline Mouth opening < 4 (cm) & 0 & 0 & - \\
\hline Mallampati score $(1 / 2 / 3)$ & $165 / 85 / 0$ & $173 / 77 / 0$ & 0.445 \\
\hline Limited neck extension & 0 & 0 & - \\
\hline Thyromental distance $<4 \mathrm{~cm}$ & 0 & 0 & - \\
\hline \multicolumn{4}{|l|}{ Characteristics of trainees } \\
\hline Number & 5 & 5 & - \\
\hline Age (year) & $29.6 \pm 1.8$ & $30.2 \pm 2.4$ & 0.003 \\
\hline Gender (male/female) & $3 / 2$ & $3 / 2$ & - \\
\hline Doctors' qualifications (years) & 2 & 2 & - \\
\hline
\end{tabular}


Table 2 Main study results and complications

\begin{tabular}{|c|c|c|c|}
\hline & T group $(n=250)$ & R group $(n=250)$ & $P$ value \\
\hline Overall intubation success rate (\%) & $237(94.8)$ & $242(96.8)$ & 0.265 \\
\hline Rate of Success at the first attempt $(n, \%)$ & $179(71.6)$ & $195(78)$ & 0.100 \\
\hline Succeed at the second attempt $(n, \%)$ & $40(16.0)$ & $36(14.4)$ & 0.301 \\
\hline Succeed at the third attempt $(n, \%)$ & $18(7.2)$ & $11(4.4)$ & 0.991 \\
\hline Failed after three attempts(n,\%) & $13(5.2)$ & $8(3.2)$ & 0.265 \\
\hline $\begin{array}{l}\text { Intubation frequency } \\
(n, \text { mean } \pm \text { SD })\end{array}$ & $\begin{array}{l}352 \\
1.4 \pm 0.7\end{array}$ & $\begin{array}{l}324 \\
1.3 \pm 0.6\end{array}$ & 0.056 \\
\hline Successful intubation time (s) & $52.8 \pm 22.2$ & $49.9 \pm 20.7$ & 0.137 \\
\hline First attempt (s) & $46.7 \pm 18.1$ & $48.6 \pm 19.9$ & 0.348 \\
\hline Second attempt (s) & $67.4 \pm 23.7$ & $52.7 \pm 22.1$ & $0.006^{*}$ \\
\hline Third attempt (s) & $80.7 \pm 19.8$ & $63.8 \pm 25.0$ & 0.054 \\
\hline \multicolumn{4}{|l|}{ Complications } \\
\hline Dry throat (\%) & $128(51.2)$ & $101(40.4)$ & $0.020^{*}$ \\
\hline Sore throat (\%) & $67(26.8)$ & $42(16.8)$ & $0.009^{*}$ \\
\hline Hoarseness (\%) & $35(14)$ & $20(8)$ & $0.045^{*}$ \\
\hline Trauma (\%) & 0 & 0 & - \\
\hline
\end{tabular}

Note: For comparison of intubation time and successful intubation time, a difference with $P$ less than 0.05 was considered statistically significant, and indicated with "*". The $P$ value was calculated using the independent-sample Student $t$ test or chi-square test. Data were expressed as mean \pm standard deviation and number (percentage)

experienced operators tended to achieve the same intubation success rate, even in difficult airways. Although the trainees received standard training before the test, they might have different learning abilities, which could lead to differences in intubation time. Thus, although the intubation time did not show any significant difference between the two groups, its clinical significance requires further investigation.

Postoperative laryngeal complications, such as sore throat and hoarseness, are common anesthesia-related complications during tracheal intubation. The frequencies of sore throat and hoarseness were reported to be $11-48 \%$ and $18-53 \%$, respectively [16-19]. In this study, no severe intubation-related complications were found, and the frequencies of sore throat and hoarseness in the two groups were consistent with previous studies. In addition, the frequencies of dry throat, sore throat, and hoarseness were found to be significantly higher in the $\mathrm{T}$ group than in the $\mathrm{R}$ group. The reason for this difference could be a lack of experience in using the intubation technique/instrument. Biro et al. revealed that differences in professional allocation and experience did not significantly affect the incidence and strength of intubation-induced laryngeal complications [20]. On the other hand, Tazeh-Kand et al. showed that the anesthesiologist experience was an independent factor influencing the frequencies of sore throat and hoarseness in male patients after intubation [18]. Recently, Inoue et al. conducted a retrospective propensity score study of 21,606 patients [21] and the frequencies of postoperative laryngeal complications were found not to be significantly different for novices and experienced anesthesiologists. Furthermore, it was believed that laryngeal complications commonly occurred after tracheal intubation, but the incidence was not associated with the experience of the operators [21]. Nevertheless, whether the incidence of complications after BIF intubation is associated with the operators' experience is still controversial and requires further investigation.

In the transillumination-assisted technique, the operator positions the BIF by finding the bright spot. A direct contact of the tube and laryngoscope tip with the laryngeal tissues leads to intubation-related damage. Although the BIF optical stylet has been proved to be safe and feasible in intubation with transilluminationassisted technique, its light source does not have a penetrability as strong as that of a Trachlight, which may be the reason for more complications in the case of the Bonfils optical stylet [7]. However, in the midline approach technique, the operator adjusts the direction and depth of the laryngoscope by observing through the eyepieces, which minimizes the direct contact of the laryngoscope tip with the vocal cords or epiglottis and other fragile tissues, hence reducing direct damage. The lightwand intubation does not rely on observing through eyepieces, and is not affected by oral secretions, blood, mist, and other factors [22]; this may have more extensive indications compared with the midline approach 
technique. However, for novices and non-difficult airway, the transillumination-assisted technique may present a higher risk of complications.

This study had the following limitations:

Premedication: Application of anticholinergic agents before anesthesia can significantly inhibit respiratory tract secretions and create favorable conditions for fiberoptic intubation. However, the common adverse reactions caused by anticholinergic agents (e.g., dry mouth) may lead to postoperative discomfort and other complications. Therefore, anticholinergic agents were not used for medication before anesthesia. However, this may hinder exposing the laryngoscope during intubation. This study was not designed to assess whether anticholinergic agents were associated with increased intubation times.

Applicability of the instrument: The difficulty in training on the use of the BIF directly leads to poor applicability of this instrument. Currently, many video laryngoscopes are available that are convenient and have a high success rate of intubation. Furthermore, many video laryngoscopes are provided with disposable laryngoscope blades, which can reduce the chance of cross-infection. Nevertheless, since the BIF has its unique scope of application and is difficult to be replaced by other laryngoscopes, it still has potential applications.

Evaluating intubation safety: The intubation time is unlikely to fully reflect the quality of intubation, while the safety and effectiveness of the operational technique are crucial. In a study by Garcia et al. [23], two layers of pressure-sensitive adhesives were applied to the conventional laryngoscope to measure the intubation force, which was considered an indicator for intubation safety and trainee's skills. However, this method is not appropriate for evaluating the BIF. In addition, the sensitivity and specificity of methods used to evaluate intubation safety still require a large number of studies. Nevertheless, the transillumination-assisted technique could be more prone to complications, especially for trainees. Therefore, we still recommend the midline approach technique group method.

Blinding: The two intubation techniques are obviously different in operation process and steps. Therefore, it is impossible to apply blinding to trachea intubation operators, observers, and data collectors. Only the patient could be blinded because they were unconscious during intubation.

Generalizability: In this study, the patients were relatively young (around 48 years old) and lean (BMI of about $22 \mathrm{~kg} / \mathrm{m}^{2}$ ). Therefore, caution should be taken when extrapolating these results to elderly, pediatric, or obese patients.

\section{Conclusion}

For a normal airway, intubation with the transilluminationassisted technique conducted by novices is unlikely to increase the success rate of intubation, but tends to increase the risk of complications. Thus, intubation with the midline approach technique could be safer for novices. The safety and efficacy for novices to manage patients with a difficult airway using the BIF requires further confirmation.

\section{Additional file}

Additional file 1: Figure S1. Linear regression analysis of intubation sequences and intubation time. Figure S2. Linear regression analysis of intubation sequences and intubation time. Figure S3. Comparison between the two groups suggests that the learning curve of the $R$ group was steeper than that of the T group. (DOCX $234 \mathrm{~kb}$ )

\section{Abbreviations}

BIF: Bonfils intubation fiberscope; BIS: Bispectral index; TOF: Train-of-four stimulation

\section{Acknowledgments}

The authors thank their anesthesiology colleagues and nurses at ShuGuang Hospital affiliated to Shanghai Traditional Chinese Medicine University for their help and patience in this study.

\section{Funding}

This work is supported by Science and Technology Commission of Shanghai Municipality (grant No. 14401932600) and National Natural Science

Foundation of China (grant No. 81471560).

\section{Availability of data and materials}

The datasets used and/or analysed during the current study available from the corresponding author on reasonable request.

\section{Authors' contributions}

JW and LY carried out the studies, participated in collecting data, and drafted the manuscript. GQF, JGS and WT performed the statistical analysis and participated in its design. GJY and FG helped to draft the manuscript. All authors read and approved the final manuscript.

\section{Competing interests}

The authors declare that they have no competing interests.

Consent for publication

Where applicable, we obtained consent for publication from the participant to report individual patient images (Fig. 1)

\section{Ethics approval and consent to participate}

This study was conducted at the Anaesthesiology Department of Shuguang Hospital Affiliated to Shanghai University of Traditional Chinese Medicine. It was approved by the ethics committee of the hospital. All participants provided written informed consent.

Received: 21 July 2016 Accepted: 13 February 2017

Published online: 21 February 2017

\section{References}

1. Benumof JL. Management of the difficult adult airway. With special emphasis on awake tracheal intubation. Anesthesiology. 1991;75:1087-110.

2. Lye ST, Liaw CM, Seet E, Koh KF. Comparison of results from novice and trained personnel using the Macintosh laryngoscope, Pentax AWS(R), C-MAC and Bonfils intubation fibrescope: a manikin study. Singapore Med J. 2013;54:64-8.

3. Piepho T, Noppens RR, Heid F, Werner C, Thierbach AR. Rigid fibrescope Bonfils: use in simulated difficult airway by novices. Scand J Trauma Resusc Emerg Med. 2009;17:33. 
4. Rudolph C, Schneider JP, Wallenborn J, Schaffranietz L. Movement of the upper cervical spine during laryngoscopy: a comparison of the Bonfils intubation fibrescope and the Macintosh laryngoscope. Anaesthesia. 2005:60:668-72.

5. Shollik NA, Ibrahim SM, Ismael A, Agnoletti V, Piraccini E, Corso RM. Use of the Bonfils Intubation Fiberscope in patients with limited mouth opening. Case Rep Anesthesiol. 2012;2012:297306.

6. Halligan $M$, Charters P. A clinical evaluation of the Bonfils Intubation Fibrescope. Anaesthesia. 2003;58:1087-91.

7. Xue FS, Liu HP, Guo XL. Transillumination-assisted endotracheal intubation with the Bonfils Fiberscope. Eur J Anaesthesiol. 2009;26:261-2.

8. Agro F, Hung OR, Cataldo R, Carassiti M, Gherardi S. Lightwand intubation using the Trachlight: a brief review of current knowledge. Can J Anaesth. 2001;48:592-9.

9. Toda J, Toda AA, Arakawa J. Learning curve for paramedic endotracheal intubation and complications. Int J Emerg Med. 2013;6:38.

10. Byhahn C, Nemetz S, Breitkreutz R, Zwissler B, Kaufmann M, Meininger D. Brief report: tracheal intubation using the Bonfils intubation fibrescope or direct laryngoscopy for patients with a simulated difficult airway. Can J Anaesth. 2008;55:232-7.

11. Corbanese U, Morossi M. The Bonfils intubation fibrescope: clinical evaluation and consideration of the learning curve. Eur J Anaesthesiol. 2009:26:622-4.

12. Wahlen BM, Gercek E. Three-dimensional cervical spine movement during intubation using the Macintosh and Bullard laryngoscopes, the bonfils fibrescope and the intubating laryngeal mask airway. Eur J Anaesthesiol. 2004;21:907-13.

13. Sui JH, Mao P, Liu JH, Tong SY, Wei LX, Yang D, et al. Transillumination-assisted orotracheal intubation: a comparison of the Bonfils fibrescope and the lightwand (Trachlight). Acta Anaesthesiol Scand. 2012;56:565-70.

14. Corbanese U, Possamai C. Re: awake orotracheal intubation with the Bonfils fiberscope in patients with a difficult airway. Eur J Anaesthesiol. 2010;27:311-2.

15. Boedeker BH, Barak-Bernhagen MA, Miller DJ, Nicholas TA, Linnaus A, Murray WB. Improving rigid fiberoptic intubation: a comparison of the Bonfils Intubating Fiberscope with a novel modification. BMC Emerg Med. 2010;10:11.

16. McHardy FE, Chung F. Postoperative sore throat: cause, prevention and treatment. Anaesthesia. 1999;54:444-53.

17. Christensen AM, Willemoes-Larsen H, Lundby L, Jakobsen KB. Postoperative throat complaints after tracheal intubation. Br J Anaesth. 1994;73:786-7.

18. Tazeh-Kand NF, Eslami B, Mohammadian K. Inhaled fluticasone propionate reduces postoperative sore throat, cough, and hoarseness. Anesth Analg. 2010;111:895-8.

19. Orandi A, Najafi A, Hajimohammadi F, Soleimani S, Zahabi S. Post-intubation sore throat and menstruation cycles. Anesth Pain Med. 2013;3:243-9.

20. Biro P, Seifert B, Pasch T. Complaints of sore throat after tracheal intubation: a prospective evaluation. Eur J Anaesthesiol. 2005;22:307-11.

21. Inoue $\mathrm{S}$, Abe R, Tanaka Y, Kawaguchi M. Tracheal intubation by trainees does not alter the incidence or duration of postoperative sore throat and hoarseness: a teaching hospital-based propensity score analysis. $\mathrm{Br} J$ Anaesth. 2015;115:463-9.

22. Falcetta S, Pecora L, Orsetti G, Gentili P, Rossi A, Gabbanelli V, et al. The Bonfils fiberscope: a clinical evaluation of its learning curve and efficacy in difficult airway management. Minerva Anestesiol. 2012;78:176-84.

23. Garcia J, Coste A, Tavares W, Nuno N, Lachapelle K. Assessment of competency during orotracheal intubation in medical simulation. $\mathrm{Br} J$ Anaesth. 2015:115:302-7.

\section{Submit your next manuscript to BioMed Central and we will help you at every step:}

- We accept pre-submission inquiries

- Our selector tool helps you to find the most relevant journal

- We provide round the clock customer support

- Convenient online submission

- Thorough peer review

- Inclusion in PubMed and all major indexing services

- Maximum visibility for your research

Submit your manuscript at www.biomedcentral.com/submit
Biomed Central 